\title{
CRISPR-Cas9-mediated pinpoint microbial genome editing aided by target-mismatched sgRNAs
}

\author{
Ho Joung Lee, ${ }^{1}$ Hyun Ju Kim, ${ }^{1}$ and Sang Jun Lee \\ Department of Systems Biotechnology, and Institute of Microbiomics, Chung-Ang University, Anseong 17546, South Korea
}

\begin{abstract}
Genome editing has been revolutionized by the CRISPR-Cas9 system. CRISPR-Cas9 is composed of single-molecular guide RNA (sgRNA) and a proteinaceous Cas9 nuclease, which recognizes a specific target sequence and a protospacer adjacent motif (PAM) sequence and, subsequently, cleaves the targeted DNA sequence. This CRISPR-Cas9 system has been used as an efficient negative-selection tool to cleave unedited or unchanged target DNAs during site-specific mutagenesis and, consequently, obtain microbial cells with desired mutations. This study aimed to investigate the genome editing efficiency of the CRISPR-Cas9 system for in vivo oligonucleotide-directed mutagenesis in bacteria. This system successfully introduced twoto four-base mutations in galK in Escherichia coli with high editing efficiencies (81\%-86\%). However, single-point mutations (T504A or C578A) were rarely introduced with very low editing efficiencies $(<3 \%)$, probably owing to mismatch tolerance. To resolve this issue, we designed one- or two-base mismatches in the sgRNA sequence to recognize target sequences in galK in E. coli. A single-point nucleotide mutation (T504A or C578A in the galKgene) was successfully introduced in 36\% $-95 \%$ of negatively selected $E$. coli cells using single-base mismatched sgRNAs. Sixteen targets were randomly selected through genome-wide single-base editing experiments using mismatched sgRNAs. Consequently, out of 48 desired single-base mutations, 25 single bases were successfully edited, using mismatched sgRNAs. Finally, applicable design rules for target-mismatched sgRNAs were provided for single-nucleotide editing in microbial genomes.
\end{abstract}

[Supplemental material is available for this article.]

The function of bacterial clustered regularly interspaced short palindromic repeats (CRISPR) is to "memorize" certain DNA sequences derived from foreign genetic elements and to degrade invasive genetic materials, such as alien plasmids and bacteriophages (Barrangou and Marraffini 2014; Westra et al. 2014). It is, therefore, referred to as a bacterial adaptive immune system. Numerous studies have investigated the biological roles and ubiquitous existence of CRISPR in bacterial genomes since the discovery of specific repeated nucleotide sequences three decades ago (Ishino et al. 1987; Mojica et al. 2000, 2005; Barrangou et al. 2007). The CRISPR/Cas system is composed of functionally modular sgRNAs and Cas proteins, which can recognize target nucleotide sequences and degrade target DNAs or RNAs. The CRISPR-Cas9 system derived from Streptococcus pyogenes, selected from among diverse CRISPR systems, is commonly used as a genome editing tool because it contains a simple complex composed of a single Cas9 polypeptide and sgRNA, which causes the double-strand breakage (DSB) of target DNAs (Makarova et al. 2015, 2018; Le Rhun et al. 2019).

Target recognition by CRISPR-Cas9 is not entirely determined by DNA-RNA hybridization between the single-stranded target DNA and target-recognizing sgRNA. The presence of protospacer adjacent motifs (PAMs) of $5^{\prime}$-NGG immediately after the target DNA $\left(\mathrm{N}_{1}-\mathrm{N}_{20}\right)$ is important for the CRISPR-Cas9 system to distinguish self- and nonself DNA (Sternberg and Doudna 2015; Moon et al. 2019). The interaction between the $5^{\prime}$-NGG PAM sequence and several amino acid residues of the Cas9 protein is critical for cleaving double-stranded target DNAs by Cas9-sgRNA complexes (Sander and Joung 2014). Because the complexity of PAM sequenc-

\footnotetext{
${ }^{1}$ These authors contributed equally to this work.

Corresponding author: sangjlee@cau.ac.kr

Article published online before print. Article, supplemental material, and publication date are at http://www.genome.org/cgi/doi/10.1101/gr.257493.119. Freely available online through the Genome Research Open Access option.
}

es may limit the availability of targetable genomic sequences (Hsu et al. 2014), other systems with alternative PAM sequences, such as CRISPR-Cpf1, have been developed and used for genome editing (Koonin et al. 2017).

The CRISPR-Cas9 system was first used to introduce precise mutations in the genomes of Streptococcus pneumoniae and Escherichia coli in combination with lambda Red recombineering (Jiang et al. 2013). When mutations are introduced into the bacterial genome via oligonucleotide-directed mutagenesis, unedited cells are expected to be eliminated by DSBs at unchanged targets by CRISPR-Cas9, and only the edited cells are expected to survive; this is called negative selection. Thus far, various CRISPR-based genome editing technologies have been developed to induce mutations, including substitution and indels across diverse bacterial species for basic genetic studies and biotechnological applications (Vento et al. 2019).

A previous study introduced a 6-bp mismatch and a 3-bp mutation in the E. coli genome with high efficiencies of 50\%-80\% (Pyne et al. 2015) and 94\%-99\% (Reisch and Prather 2015), respectively, through lambda Red-mediated recombineering of oligonucleotides, followed by CRISPR-Cas9 negative selection. Because the lambda Bet protein promotes recombineering of single-stranded DNA (Costantino and Court 2003), Bet-mediated recombineering along with CRISPR-Cas9 facilitated genomic editing of two to three bases in galK, $x y l A$, and lacZ in $E$. coli with very high efficiencies (96.5\%-99.7\%) (Ronda et al. 2016). However, single-base editing is uncommon, probably owing to the mismatch tolerance of CRISPR-Cas9, which might recognize the single-base edited sequence as the target.

In this study, to obtain single-base edited cells, we designed one or two mismatched sequences in the target recognition

(C) 2020 Lee et al. This article, published in Genome Research, is available under a Creative Commons License (Attribution 4.0 International), as described at http://creativecommons.org/licenses/by/4.0/. 
sequences of sgRNAs. To determine whether negative selection using the mismatched sgRNAs is effective, various single-point mutations creating stop codons in galK were tested as the target of CRISPR-Cas9 in E. coli. Furthermore, the number of mismatched base pairs in the PAM distal or proximal regions was assessed. Moreover, genome-wide single-base editing experiments were performed to clarify design rules for mismatched sgRNAs for successful negative selection.

\section{Results}

\section{Generation of multiple-base mutations through CRISPR-Cas9 negative selection}

Mutagenic oligonucleotides introducing stop codons (C168Z) in galK and sgRNA plasmids were electroporated into both Cas9 nuclease-overexpressing and lambda Bet protein-overexpressing $E$. coli MG1655 cells. If galK mutations were generated as intended, white colonies with the corresponding $\mathrm{Gal}^{-}$phenotype would be observed on MacConkey agar plates owing to premature translation termination of the nascent galactokinase protein (Fig. 1). The white and red colonies were enumerated to determine the genome editing efficiency. Cas9/sgRNA complexes can recognize the target $\left(\mathrm{N}_{20}\right)$ sequence $\left({ }^{498}\right.$ AGGCTGTAACTGCGGGATCA ${ }^{517}$ in the galK gene) and generate DNA DSBs in the galK target, ultimately eliminating the unedited cells.

Consequently, two- to four-base substitutions $\left({ }^{504} \mathrm{TA}\right.$ to AT, ${ }^{503}$ GTA to AGC, and ${ }^{504}$ TAAC to ATCA) were successfully intro-
A

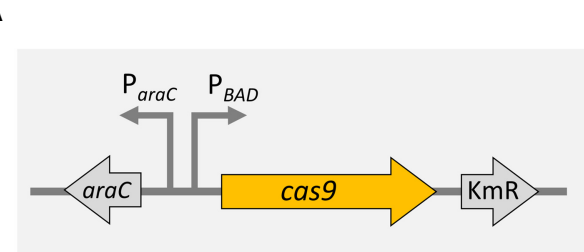

B

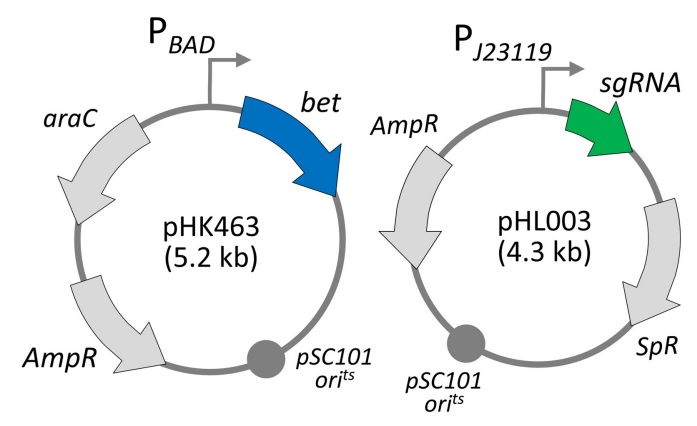

C
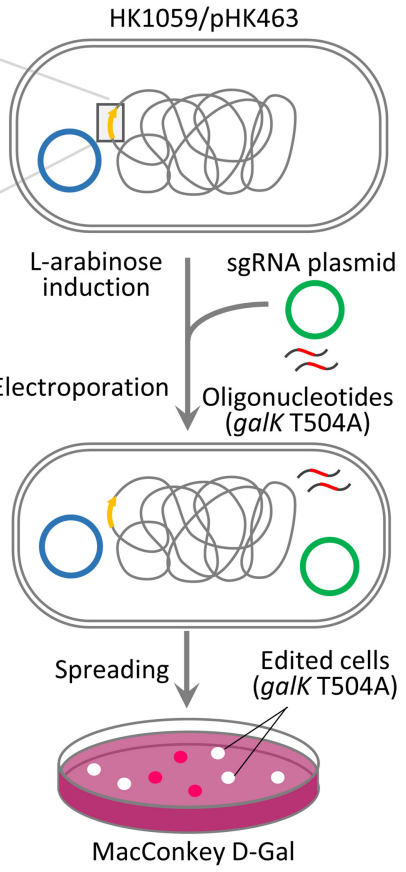

Figure 1. Schematic representation of bacterial genome-editing. (A) Chromosomal construction of cas9 downstream from the $\mathrm{P}_{B A D}$ promoter, which forms an L-arabinose-inducible cas 9 gene. (B) Construction of plasmids. Lambda bet expression vector (pHK463) was derived from pKD46 plasmid. The sgRNA expression plasmid ( $\mathrm{pHL003)}$ harbors a temperature-sensitive origin for iterative genome engineering. (C) Genome editing. Mutagenic oligonucleotides carrying galK T504A and sgRNA plasmids were electroporated into $E$. coli cells overexpressing Cas9 and Bet proteins by L-arabinose induction. Recovered cells were spread on MacConkey agar containing D-galactose. Red/white colonies were counted for determination of galK editing efficiency. duced, with editing efficiencies of $81 \%-86 \%$ (Fig. 2). However, single-point mutations $\left({ }^{504} \mathrm{~T}\right.$ to A) were rarely introduced with very low editing efficiencies (2\%) in galK. These results indicate that even single-base mutated DNA targets may be recognized as unchanged DNA targets and digested by the Cas9/sgRNA complex in cells; this is referred to as the mismatch tolerance of the CRISPR-Cas9 system (Lin et al. 2014; Fu et al. 2016).

In the absence of mutagenic oligonucleotides, all unedited cells would be expected to be eliminated by CRISPR-Cas9. However, numerous colonies survived ( $\left.>10^{3} \mathrm{CFU} / \mu \mathrm{g}\right)$, probably owing to the existence of a subpopulation (approximately one out of $10^{5}$ cells) of cells wherein the CRISPR-Cas9 is not functional.

\section{Single-base genome editing with target-mismatched sgRNAs}

Based on the successful results of multiple substitutions, we genererance (Fig. 3A). DNA targets harboring single-base substitutions were presumed to not be recognized by Cas9/target-mismatched complexes. The sgRNA target recognition sequence was designed to harbor one or two mismatches located contiguously, flanking the point mutagenic base $\left({ }^{504} \mathrm{~T}\right)$ in the mutagenic oligocorrectly in the genome, two mismatches can be generated between one base-mismatched sgRNAs and single-base mutated tar-

Consequently, a single-point mutation (T504A) in the galK gene was successfully introduced using single-base mismatched sgRNAs. When using sgRNA (A8C), the editing efficiency was $95 \%$, determined as the number of white colonies formed by a nonsense galK mutation (T504A) divided by the total number of white and red colonies formed on MacConkey agar plates containing D-galactose and spectinomycin (Fig. 3C). Ten white colonies were selected, and the T504A mutation in galK was confirmed through Sanger DNA sequencing. In the case of sgRNA (G6C), white colonies were obtained with a decreased editing efficiency (36\%). Furthermore, double-base mismatched sgRNAs (A8C/A9C and T5G/G6C) were assessed. With sgRNA (A8C/A9C), a single-point mutation (T504A) was introduced with $86 \%$ efficiency. However, when another sgRNA (T5G/G6C) was used, we observed no white colonies on the MacConkey plate, indicating unsuccessful negative selection. The survival rate for T5G/G6C was increased to $3.4 \times$ $10^{7} \mathrm{CFU} / \mu \mathrm{g}$ DNA, presumably because the Cas9/sgRNA (T5G/G6C) complex cannot appropriately generate DSBs at the unchanged target DNAs in the unedited cells.

\section{Pinpoint genome editing by target- mismatched sgRNAs in the PAM proximal region}

Base-paring between sgRNA and the target DNA in the PAM proximal region is 
A

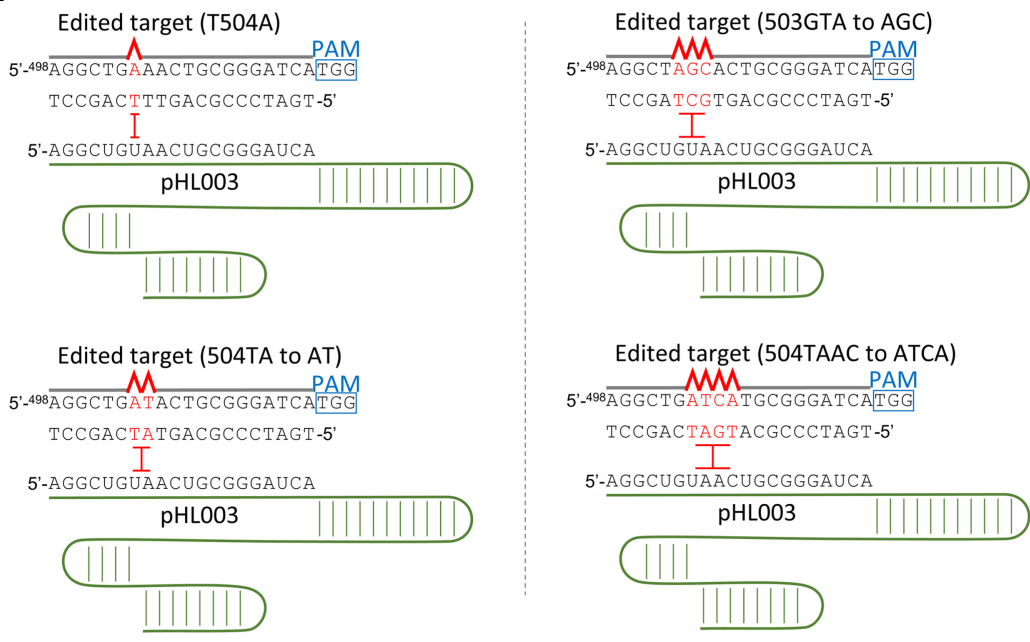

B

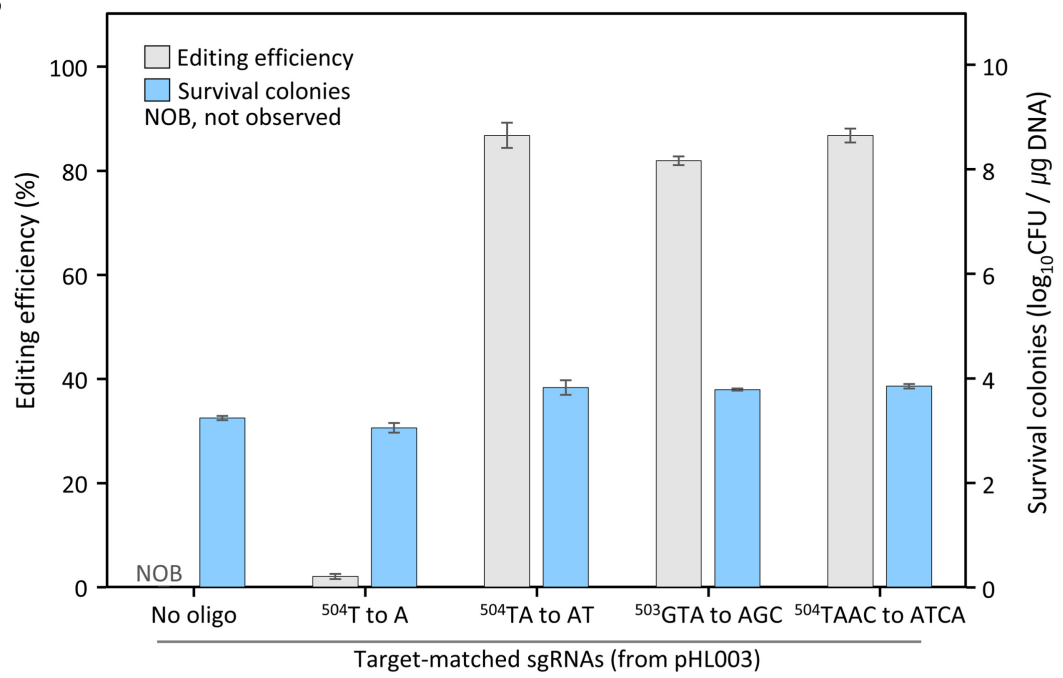

C
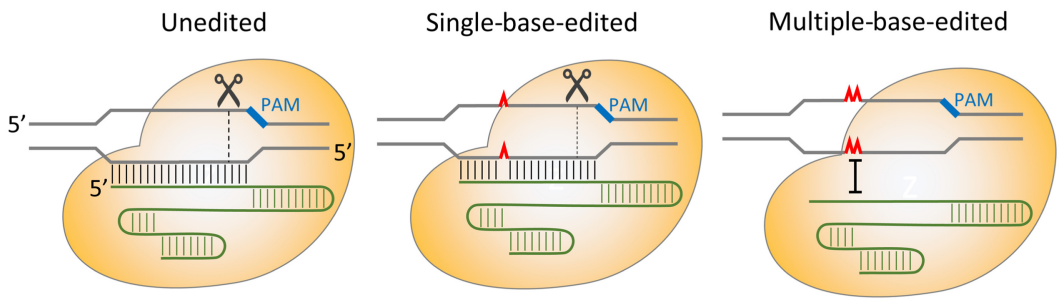

Figure 2. Genome editing efficiency of oligonucleotide-directed mutagenesis followed by Cas9/ sgRNA-mediated negative selection. (A) Design of target-matched sgRNAs for negative selection of edited targets in galK. (B) The editing efficiency of the galK mutation using single-, double-, triple-, and quadruple-base mutagenic oligonucleotides. Each bar represents the mean of three independent experiments. (C) Cleavage of both unedited and single-base-edited targets by CRISPR-Cas9 owing to mismatch tolerance.

more critical for target recognition and cleavage by CRISPR-Cas9 (Cencic et al. 2014; Anderson et al. 2015). Therefore, proximal mismatches between sgRNA and the target DNA were assessed herein (Fig. 4A). In the case of target-matched sgRNA for single-point mutation (C578A) in the galK gene, we obtained some white colonies $(<2.3 \%)$. In the case of single-base mismatched sgRNAs (A17C and
T15G), the editing efficiencies were $84 \%$ and $82 \%$, respectively, and the number of surviving cells was $<10^{4} \mathrm{CFU} / \mu \mathrm{g}$ DNA. However, in the case of doublemismatched sgRNAs (A17C/C18A, and C14A/T15C), no white colonies were observed and survival rates were markedly higher $\left(\geq 10^{6} \mathrm{CFU} / \mu \mathrm{g}\right.$ DNA), indicating that Cas9/double base-mismatched sgRNA complexes cannot recognize the unedited targets (Fig. 4B). These results show that single-base mismatched sgRNAs allow efficient negative selection, regardless of mismatches in the PAM proximal or distal region.

\section{Plasmid curing and deletion of cas9 for scar-free genome engineering}

All sgRNA plasmids harbor a temperature-sensitive origin (pSC101 ts-ori) that can be cured through incubation at $42^{\circ} \mathrm{C}$, enabling iterative genome editing using different oligonucleotides and sgRNA plasmids. After genome editing, genome-integrated cas9 was replaced with the original $a r a B A D$ gene through P1 transduction, and the transformed cells were selected in L-arabinose minimal medium and confirmed through selection in MacConkey agar containing D-galactose or L-arabinose (Fig. 5). Consequently, scar-free single-base substitutions could be achieved in bacterial genomes.

\section{Genome-wide single-base editing using single mismatched sgRNAs}

To generate applicable design rules for mismatched sgRNAs, 16 target sequences for CRISPR-Cas9 negative selection were randomly selected in the genome of $E$. coli. Base-editing sites were designed at $\mathrm{N}_{11}$ in target sequences, except $\mathrm{N}_{7}$ in galK (504) and $\mathrm{N}_{16}$ in galK (578), in 48 mutagenic oligonucleotides (three oligonucleotides per target, i.e., $\mathrm{A} \rightarrow \mathrm{G} / \mathrm{T} / \mathrm{C}$, $\mathrm{T} \rightarrow \mathrm{G} / \mathrm{A} / \mathrm{C}, \mathrm{G} \rightarrow \mathrm{A} / \mathrm{T} / \mathrm{C}$, and $\mathrm{C} \rightarrow \mathrm{G} / \mathrm{A} / \mathrm{T}$ ) (Supplemental Table S3). Because singlebase mismatched sgRNAs accurately edited galK (Figs. 3, 4), three sgRNAs with different single-base mismatches (immediately adjacent to edited bases, i.e., $\mathrm{N}_{12}^{\prime}: \mathrm{N}^{\prime \prime}{ }_{12}$ ) in each target were used for negative selection (Fig. 6A; Supplemental Fig. S1). As a control, target-matched sgRNAs were used for each target (Supplemental Table S5). Therefore, 192 combinatorial electroporations ( 16 targets $\times 3$ mutagenic oligonucleotides $\times 4$ sgRNAs/target) were performed, and subsequent base editing in three randomly selected colonies (per target) was analyzed via PCR followed by Sanger sequencing (Supplemental Fig. S2). One,

\section{Genome Research}

www.genome.org 
A

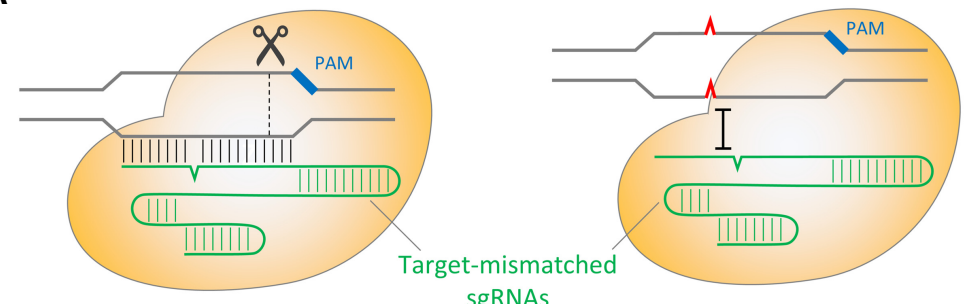

B
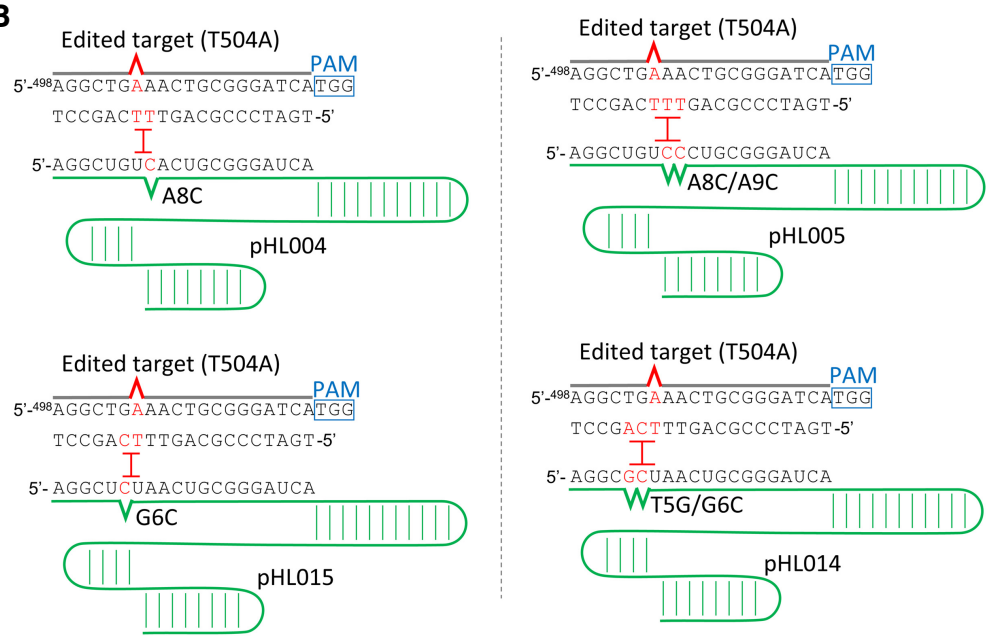

C

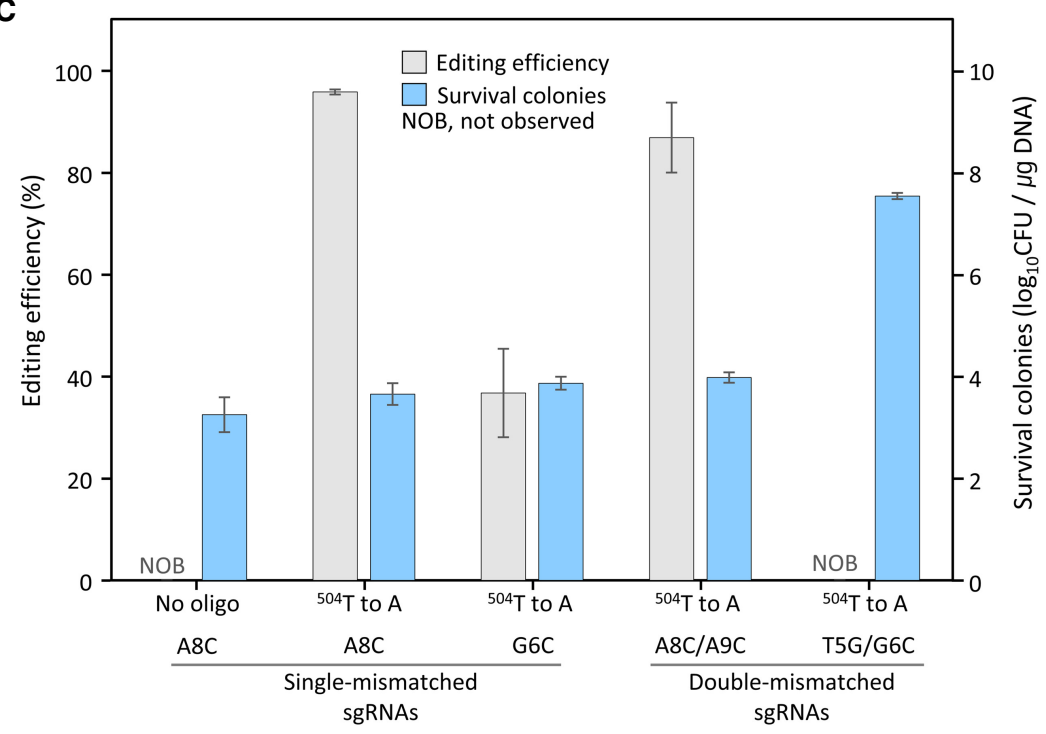

Figure 3. Negative selection of single-base-edited DNA targets aided by target-mismatched sgRNAs via CRISPR-Cas9. (A) Mismatch tolerance allows unedited targets to be cleaved by the Cas9/target-mismatched sgRNA complex. Single-base-edited targets cannot be recognized by the Cas9/target-mismatched sgRNA complex owing to multiple mismatches. (B) Design of target-mismatched sgRNAs for single-base editing (T504A) in galK. Single or double mismatch(es) were located adjacent to the T504A point mutation site. (C) Single-base genome editing efficiencies using single- or double-base mismatched sgRNAs and the number of surviving cells. Each bar represents the mean of three independent experiments.

two, or three edited sequences from the three reactions (in each electroporation) were considered successful base editing.

Consequently, of 48 types of single-base editing in 16 targets (equivalent to the number of mutagenic oligonucleotides), 25 types of base changes in 13 targets were successfully obtained using mismatched sgRNAs $\quad(52 \%=25 / 48) \quad$ (Supplemental Table S6). To determine which mutations are effectively introduced by which mismatched sgRNAs, we analyzed 41 individual single-base editings (among 192 electroporated samples) for the type of base editings and mismatch patterns between DNA targets and sgRNAs.

First, we analyzed the data by baseediting types (64 transitions and 128 transversions). Among 41 single-base editings, transition changes were rarely observed $(8 \% ; 5 / 64)$. However, transversion base editings were more frequent (28\%; 36/128). Among 41 cases, only one was obtained through targetmatched sgRNAs. Transition at $\mathrm{N}_{11}$ resulted in moderate base mispairing, including Pu:Py or Py:Pu between $\mathrm{N}^{\prime}{ }_{11}$ in a non-PAM strand and $\mathrm{N}^{\prime \prime}{ }_{11}$ in an sgRNA; however, transversion at $\mathrm{N}_{11}$ caused base mispairing such as $\mathrm{Pu}: \mathrm{Pu}$ or Py:Py at $\mathrm{N}_{11}^{\prime}: \mathrm{N}^{\prime \prime}{ }_{11}$.

Because base editing is apparently affected by mismatch patterns between $\mathrm{N}_{11}^{\prime}$ and $\mathrm{N}^{\prime \prime}{ }_{11}, 41$ base-editing cases were analyzed on the basis of mispairing patterns between $\mathrm{N}_{12}^{\prime}$ and $\mathrm{N}^{\prime \prime}{ }_{12}$ (Fig. 6B). In cases of transversions, $43.7 \%$ of singlebase editings were obtained with Py:Py and $\mathrm{Pu}: \mathrm{Pu}$ mispairings at $\mathrm{N}_{12}^{\prime}: \mathrm{N}^{\prime \prime}{ }_{12}$. Less efficient base editings were observed in Py:Pu (15.6\%) and Pu:Py (9.3\%), wherein target-matched sgRNAs were used in half of the electroporation samples (32/64). In cases of transition, no base editing was observed with Py:Pu and Pu:Py at $\mathrm{N}_{12}^{\prime}: \mathrm{N}^{\prime \prime}{ }_{12}$. However, Py:Py and Pu:Pu mismatches facilitated singlebase editing with $6.3 \%$ and $25 \%$ efficiencies, respectively. These data indicate that two consecutive mismatches of Py: Py or Pu:Pu between non-PAM strands and sgRNAs appear efficient in singlebase editing aided by target-mismatched sgRNAs in CRISPR-Cas9.

\section{Discussion}

As the CRISPR-Cas9 technology has accelerated and simplified accurate genetic engineering in cells, genomic mutations occurring either spontaneously during replication or generated in vitro can be rectified. An important goal of genome editing in biotechnology applications is to introduce desired mutations into new backgrounds for directed genetic engineering of useful phenotypes (Bailey et al. 1996).

DNA DSBs caused by CRISPR-Cas9 can be restored through nonhomologous end joining (NHEJ), which can inactivate target 
A

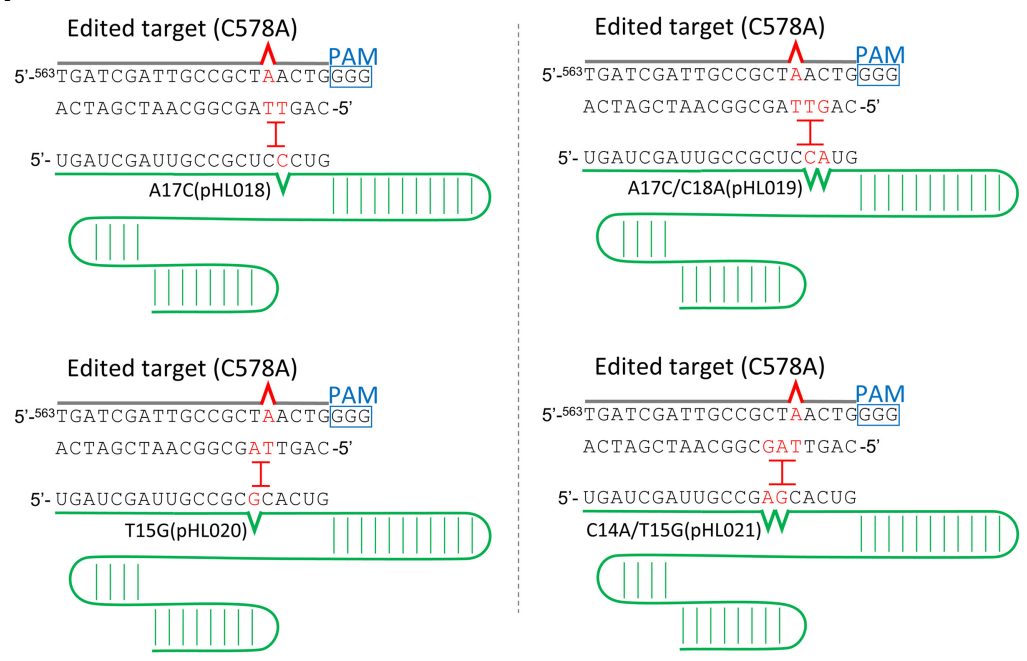

B

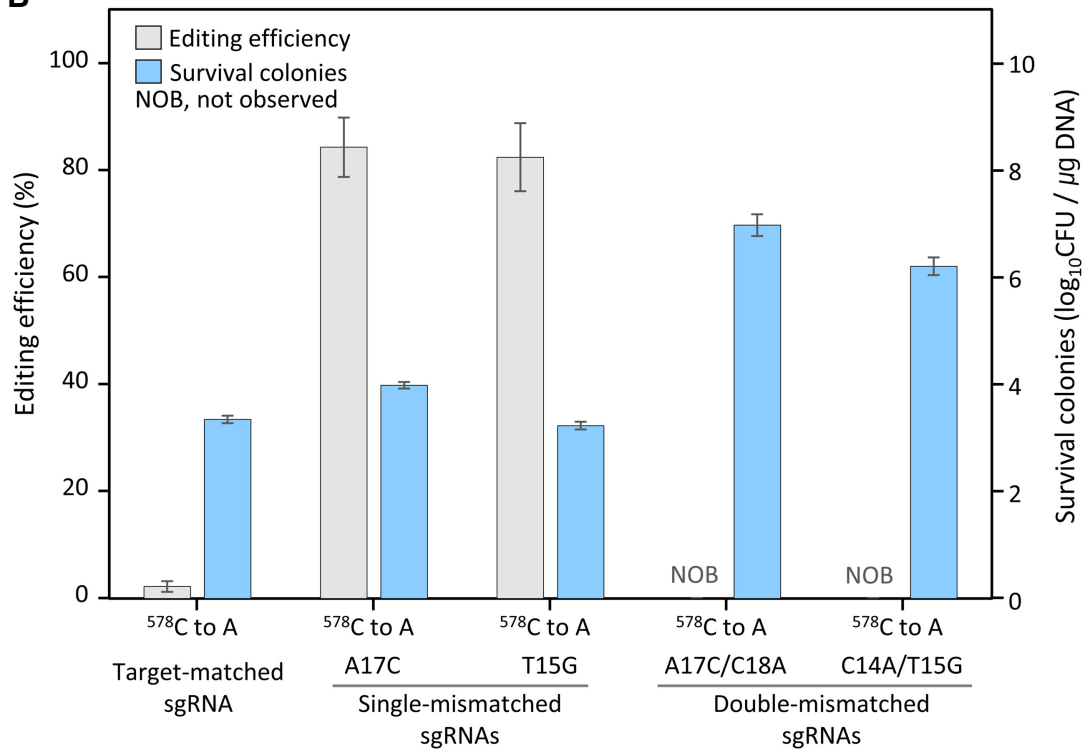

Figure 4. Single-base editing in the PAM-proximal region using target-mismatched sgRNAs. $(A)$ Negative selection of edited target sequence (C578A in the galK gene) by Cas9/target-mismatched sgRNA complexes. Single or double mismatch(es) were located adjacent to the C578A point mutation site. (B) Genome editing efficiencies using target-matched, single- or double-base mismatched sgRNAs and the number of surviving cells. Each bar represents the mean of three independent experiments.

genes through indel or frame-shift mutations. If homologous regions flanking double-stranded DNA or oligonucleotides are concomitantly added, cells harboring unchanged target sequences can be eliminated via CRISPR-Cas9, and edited cells can survive because of the lack of a target site (Mougiakos et al. 2016; Ronda et al. 2016).

Oligonucleotide-directed mutagenesis combined with CRISPR-Cas9 has been developed for convenient editing of bacterial genomes (Jiang et al. 2013; Pyne et al. 2015). However, the introduction of single-point mutations into bacterial genomes by CRISPR-Cas9 has been only rarely observed. Our experiments have shown that single-point genomic mutations cannot be obtained through negative selection using CRISPR-Cas9 (Fig. 2).
Four different mutagenic oligonucleotides were electroporated into E. coli cells with the same sgRNA plasmid. Two- to four-base substitutions were obtained with high efficiency $(81 \%-86 \%)$. However, no single-base mutated cell was obtained among several hundred surviving cells. This may have resulted because single-point mutations seem to be recognized as the DNA targets owing to mismatch tolerance of CRISPR-Cas9 (Jinek et al. 2012; Liang et al. 2017).

The CRISPR-Cas9 system originally evolved as a bacterial adaptive immune system. CRISPR-Cas9 has been considered to degrade external nonself DNAs from phages or mobile DNAs, notwithstanding single mutations in the target DNA or target-specific sgRNA sequences. Because the number of redundant permutations $\left(\sim 4^{20}\right)$ of possible target sequences, other than the PAM sequence, is markedly greater than the size of bacterial genomes $\left(\sim 4^{11}\right)$, one or two mismatches are still safe and tolerant to distinguish self and nonself DNA. Thus, mismatch tolerance involving CRISPR-Cas9 is inevitable and a natural consequence of evolution.

Based on our results indicating the lack of single-base mutations and because multiple-base substitution mutations are successfully obtained with high efficiency, we designed and introduced target-mismatched sequences in sgRNAs in advance so as not to recognize single-base mutated sequences as the target (Fig. 3A). Because oligonucleotide-directed mutagenesis alters at least two successive bases (Fig. 2A), one or two mismatched sequences in sgRNAs were required on both sides of a single-base substitution. Single-base mismatched sgRNAs helped us induce single-point mutations (Fig. 3C). Our results indicate that single-base mismatched sgRNAs facilitate efficient negative selection, regardless of mismatches in the PAM-proximal or PAM-distal regions (Figs. 3, 4). Moreover, among four different double-base mismatched sgRNAs (A8C/A9C, T5G/G6C, A17C/C18A, and C14A/T15G), only sgRNA (A8C/A9C) facilitated negative selection of single-point mutations, indicating that accurate negative selection cannot be expected using double-base mismatched sgRNAs.

During genome-wide single-base editing, some genes are more susceptible to base editing than others. We observed high single-base editing efficiencies (four or more of nine) with mismatched sgRNAs in four targets: mnmE, fau, proX, and $y d c O$. However, no single-base editing was observed in three DNA targets: galK (934), $y p d A$, and $y j h F$ genes (Supplemental Table S6). In galK, several edited bases were obtained in galK (504) and (578) targets but not in galK (934), indicating that the efficiency

\section{Genome Research}

www.genome.org 
A

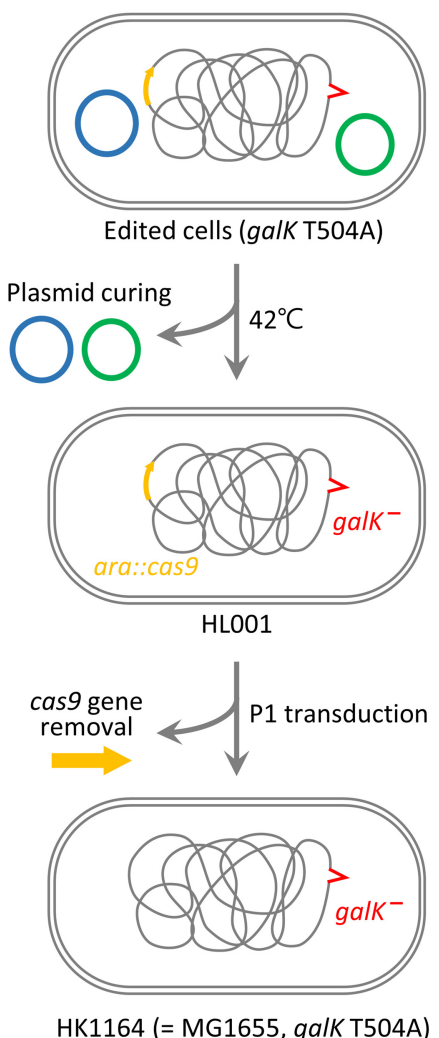

B
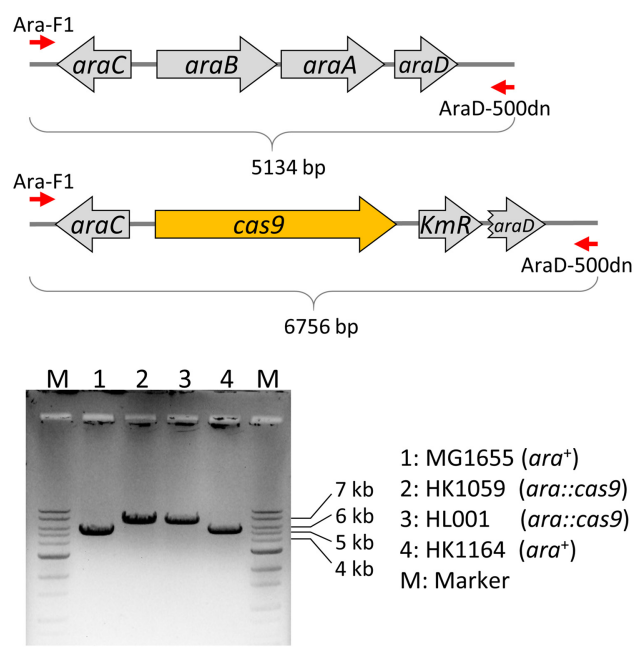

C

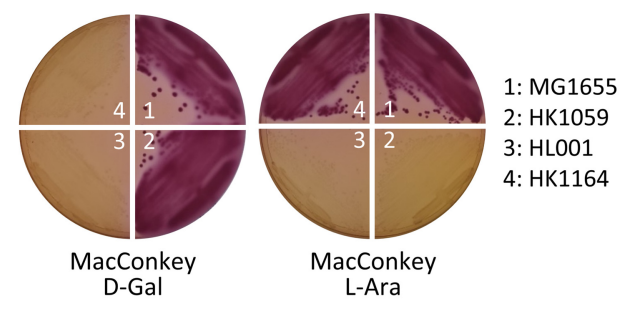

Figure 5. Scar-free single-base genome engineering. (A) Plasmids in the edited cells were cured through incubation at a high temperature, and the cas9-KmR cassette was replaced with the $\operatorname{araBAD}$ operon through P1 transduction. (B) The chromosomal structures in the ara operon in E. coli MG1655, HK1059, HL001, and HK1164 cells were confirmed via PCR. (C) The phenotype (Gal ${ }^{-} \mathrm{Ara}^{+}$) of singlebase-edited HK1164 cells (=MG1655, galK T504A), as confirmed through cell culture on MacConkey agar supplemented with D-galactose or L-arabinose.

of base editing may have been affected by target DNA sequences rather than the chromosomal location.

In the single-base editing experiments, transversion base changes were easily obtained, causing Py:Py or Pu:Pu at $\mathrm{N}^{\prime}{ }_{11}$ : $\mathrm{N}^{\prime \prime}{ }_{11}$ between non-PAM strands and sgRNAs, when adjacent Py: Py or Pu:Pu at $\mathrm{N}_{12}^{\prime}: \mathrm{N}^{\prime \prime}{ }_{12}$ was designed in mismatched sgRNAs for negative selection (Fig. 6). Two consecutive mismatches potentially result in inaccurate conformations of DNA (non-PAM strand) and RNA (sgRNA) duplexes, potentially misleading target recognition. Even one mismatch (Py:Py or Pu:Pu) could be better recognized as targets by CRISPR-Cas9. For G:U wobble base-pairing (Varani and McClain 2000), only one single-base editing was successful in 12 cases (Supplemental Table S6). Consequently, G:U base-pairing was not effective for negative selection. Although the sgRNA plasmid was successfully constructed, negative selection failed in six electroporation samples, wherein more than $10^{7}$ survival colonies (CFU/ $\mu$ g) were obtained.

Considering the design of target-mismatched sgRNAs for single-base editing of microbial genomes, it is important to consider the following points: (1) Transversion editing is more effective, (2) there is Py:Py or Pu:Pu base mispairing between non-PAM strands and sgRNAs, and (3) the G:U base pair is not markedly effective.

In summary, a single-base microbial genome editing method was developed from the use of mismatch tolerance, which has been an obstacle in the negative selection of unedited targets by CRISPR-Cas9 system. Target-mismatched sgRNA methods can be used for accurate genome reprogramming, including the fine-tuning of promoter strength and editing of codons of interest in microbial cells.

\section{Methods}

\section{Strains and culture conditions}

E. coli strains used herein are listed in Supplemental Table S1 and were grown in Luria-Bertani broth (LB) at $30^{\circ} \mathrm{C}$ or $37^{\circ} \mathrm{C}$, depending on the plasmid ori sequence. E. coli DH5 $\alpha$ and MG1655 were used as cloning hosts and for genomic integration of cas9. E. coli MG1655 cells harboring plasmid pKD46 were grown in $\mathrm{LB}$ containing ampicillin $(50 \mu \mathrm{g} / \mathrm{mL})$ at $30^{\circ} \mathrm{C}$, and L-arabinose (final $1 \mathrm{mM}$ ) was added when the optical density at $600 \mathrm{~nm} \quad\left(\mathrm{OD}_{600}\right)$ approached 0.4 to up-regulate lambda recombinases. Subsequently, electrocompetent cells were harvested, washed, resuspended in $10 \%$ glycerol solution, and stored at $-80^{\circ} \mathrm{C}$ until electroporation for recombineering. The construction of an E. coli strain carrying the cas 9 gene in the chromosome is described below. When needed, kanamycin and spectinomycin were added to the culture medium at 25 and $75 \mu \mathrm{g} / \mathrm{mL}$, respectively.

\section{Genomic integration}

Primers used to construct E. coli HK1059 are listed in Supplemental Table S2. cas 9 was PCR-amplified using the plasmid pCas (a gift from Sheng Yang; Addgene plasmid 62225) as a template and was fused with a kanamycin-resistance marker through splice-overlap PCR to generate a cas9-KmR cassette. The cas9-KmR cassette was amplified with primer pairs harboring homologous DNA sequences for recombineering, and subsequently, the purified PCR products were electroporated into L-arabinose-induced E. coli MG1655 harboring plasmid pKD46 for genomic integration of the cas 9 gene in the arabinose operon. Finally, cas 9 was located downstream from the L-arabinose-inducible $\mathrm{P}_{B A D}$ promoter on the chromosome of MG1655. The strain was designated as E. coli HK1059.

\section{Plasmid construction}

Plasmids used for base editing of galK are listed in Supplemental Table S1. The bet gene, and the pKD46 backbone excluding three lambda recombinase genes exo, bet, and gam, were amplified via PCR. These two fragments were assembled by isothermal assembly using a Gibson assembly master mix (NEB) to generate plasmid pHK463, which could only express Bet proteins after L-arabinose induction.

sgRNA expression plasmid vectors were constructed as follows. The galK in E. coli was selected as a target gene for editing. We amplified DNA fragments containing an ampicillin-resistance gene and the temperature-sensitive origin of replication in the 
A

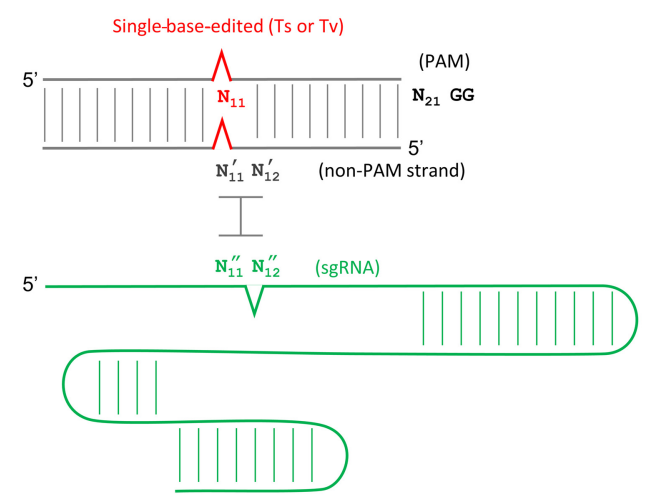

B

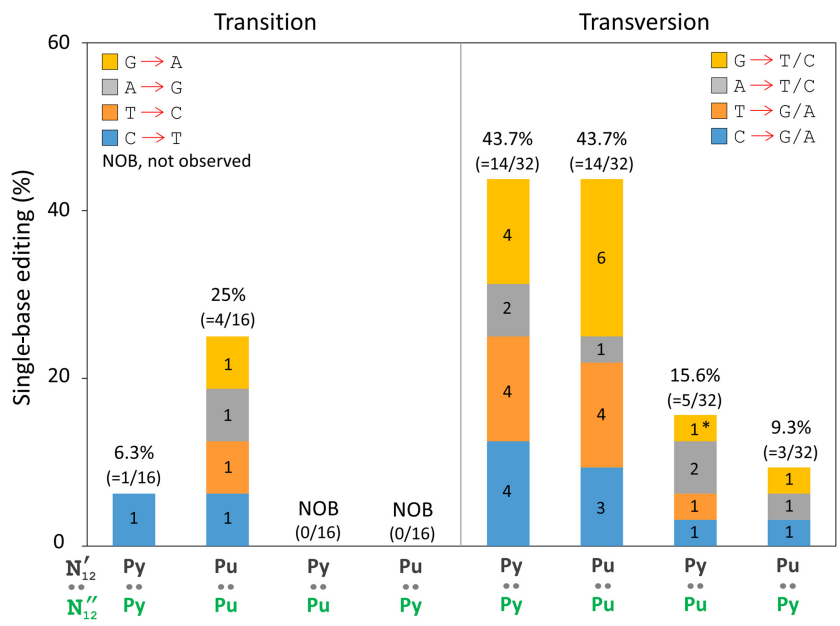

Figure 6. Genome-wide single-base editing aided by target-mismatched sgRNAs in CRISPR-Cas9. (A) Design of mismatched sgRNAs for single-base-edited targets. Single mismatches $\left(\mathrm{N}_{12}^{\prime}: \mathrm{N}^{\prime \prime}{ }_{12}\right)$ between non-PAM strands and sgRNAs were introduced immediately after the single-base-edited sites $\left(\mathrm{N}_{11}\right)$. Note that mismatches in two galK ([504] and [578]) targets were designed at $\mathrm{N}_{8}^{\prime}: \mathrm{N}^{\prime \prime}{ }_{8}$ and $\mathrm{N}_{17}: \mathrm{N}^{\prime \prime}{ }_{17}$, respectively. (B) Single-base genome editing for 16 different DNA targets was aided by various base-parings $\left(\mathrm{N}^{\prime}{ }_{12}: \mathrm{N}^{\prime \prime}{ }_{12}\right)$. Numbers in parentheses and colored boxes are the frequency and the successful events of single-base editing, respectively. Asterisk indicates one single-base editing was observed using a target-matched sgRNA among 16 targetmatched sgRNAs.

pKD46 plasmid. The spectinomycin-resistance gene and the sgRNA gene were amplified using pTargetF (a gift from Sheng Yang; Addgene plasmid 62226) as a template. Two fragments were digested with $\mathrm{ClaI}$ and $\mathrm{NcoI}$ endonucleases and ligated to each other to form pHL001. Other sgRNA plasmids (Supplemental Table S1) were generated through Gibson assembly, using pHL001 as a template.

\section{Base editing in galK}

E. coli HK1059 harboring pHK463 was cultured in LB supplemented with ampicillin $(50 \mu \mathrm{g} / \mathrm{mL})$ at $30^{\circ} \mathrm{C}$, and L-arabinose (final 1 $\mathrm{mM}$ ) was added at an $\mathrm{OD}_{600}$ of 0.4 to up-regulate Cas9 nucleases and lambda Bet proteins for oligonucleotide-directed mutagenesis. Thereafter, the cells were prepared and stored at $-80^{\circ} \mathrm{C}$ for subsequent electroporation of mutagenic oligonucleotides and sgRNA plasmids. Mutagenic oligonucleotides (Supplemental Table S2) were designed to introduce a multiple-base substitution (TA504AT, GTA503AGC, or TAAC504ATCA) or single-base substitution (T504A or C578A), each introducing a premature stop codon in galK. For negative selection of mutant cells harboring single-base substitutions, mismatched sgRNA plasmids were designed to harbor one or two mismatches with the target $\left(\mathrm{N}_{20}\right)$. The mismatches were located adjacent to the point mutation sites (Figs. 3B, 4A).

Each mutagenic oligonucleotide (100 pmol), with sgRNA plasmids (200 ng), was electroporated in E. coli HK1059 cells harboring pHK463 to introduce one- to four-base substitutions and for CRISPR-Cas9-mediated negative selection. Electroporation was performed at $25 \mu \mathrm{F}, 200 \Omega$, and $1.8 \mathrm{kV}$, and a $0.1-\mathrm{cm}$ electroporation cuvette was used. Immediately thereafter, the cells were transferred to $950 \mathrm{~mL}$ of SOC and incubated at $30^{\circ} \mathrm{C}$, at $180 \mathrm{rpm}$ for $1 \mathrm{~h}$ for recovery, and then spread on MacConkey agar containing D-galactose $(0.5 \%)$ and spectinomycin $(75 \mu \mathrm{g} / \mathrm{mL})$ and incubated for $24 \mathrm{~h}$ at $30^{\circ} \mathrm{C}$. The genome editing efficiencies of oligodirected mutagenesis of $\mathrm{galK}$ followed by CRISPR-Cas9 negative selection were calculated by counting white and red colonies expressing D-galactose-fermenting phenotypes (Costantino and
Court 2003). Surviving cells were enumerated to confirm whether target digestion by the CRISPR-Cas9 system was efficient.

\section{Elimination of the editing tool}

After editing, cas9 nuclease was replaced with the original $\operatorname{araBAD}$ operon through P1 transduction. To transfer the $\operatorname{araB}$, araA, and araD genes of the MG1655 strain to an HK1059 background, we used P1 lysates of MG1655 to transduce HK1059 recipient cells (Fig. 5A). Subsequently, transduced cells were selected on M9 minimal medium containing $0.4 \% \mathrm{~L}$-arabinose. Gene replacement by P1 transduction was confirmed via PCR (Fig. 5B).

\section{Genome-wide single-base editing}

Sixteen targets including three targets in galK were randomly selected in the E. coli genome, and single-base mutagenic oligonucleotides for 16 targets are listed in Supplemental Table S3. sgRNA plasmids for these 16 targets were constructed through Gibson assembly, using pTargetF harboring pBR322 ori as a template and primers listed in Supplemental Table S4. Finally, plasmids expressing target-matched sgRNAs and single mismatched sgRNAs are listed in Supplemental Table S5.

For genome-wide single-base editing experiments, each single-base mutagenic oligonucleotide ( $100 \mathrm{pmol})$ and corresponding sgRNA plasmid (200 ng) were electroporated into L-arabinose-induced HK1059 cells harboring pHK463. Electroporated samples were spread on LB agar plates containing spectinomycin $(75 \mu \mathrm{g} /$ $\mathrm{mL}$ ). After $20 \mathrm{~h}$ at $37^{\circ} \mathrm{C}$, three colonies (per electroporation) were randomly selected, and Sanger sequencing was performed to confirm the desired base editing in the bacterial genome using primers listed Supplemental Table S4.

\section{Competing interest statement}

H.J.L., H.J.K., and S.J.L. have filed a patent application based on this work. 


\section{Acknowledgments}

We thank Siheon Kim and Juan Park at our department for their technical assistance. This study was supported by the National Research Foundation of Korea (NRF-2017R1E1A1A01075124 and 2019R1A4A1024764) and research grants from the CJ CheilJedang Institute of Biotechnology (CG-20-17-01-0002) and the Korea Research Institute of Bioscience and Biotechnology (KRIBB) research initiative program, Republic of Korea.

\section{References}

Anderson EM, Haupt A, Schiel JA, Chou E, Machado HB, Strezoska Ž, Lenger S, McClelland S, Birmingham A, Vermeulen A, et al. 2015. Systematic analysis of CRISPR-Cas9 mismatch tolerance reveals low levels of off-target activity. J Biotechnol 211: 56-65. doi:10.1016/j.jbiotec.2015 .06 .427

Bailey JE, Sburlati A, Hatzimanikatis V, Lee K, Renner WA, Tsai PS. 1996. Inverse metabolic engineering: a strategy for directed genetic engineering of useful phenotypes. Biotechnol Bioeng 52: 109-121. doi:10.1002/ (SICI)1097-0290(19961005)52:1<109::AID-BIT11>3.0.CO;2-J

Barrangou R, Marraffini LA. 2014. CRISPR-Cas systems: prokaryotes upgrade to adaptive immunity. Mol Cell 54: 234-244. doi:10.1016/j.molcel.2014 .03 .011

Barrangou R, Fremaux C, Deveau H, Richards M, Boyaval P, Moineau S, Romero DA, Horvath P. 2007. CRISPR provides acquired resistance against viruses in prokaryotes. Science 315: 1709-1712. doi:10.1126/sci ence. 1138140

Cencic R, Miura H, Malina A, Robert F, Ethier S, Schmeing TM, Dostie J, Pelletier J. 2014. Protospacer adjacent motif (PAM)-distal sequences engage CRISPR Cas9 DNA target cleavage. PLoS One 9: e109213. doi:10 .1371/journal.pone.0109213

Costantino N, Court DL. 2003. Enhanced levels of $\lambda$ Red-mediated recombinants in mismatch repair mutants. Proc Natl Acad Sci 100: 1574815753. doi:10.1073/pnas.2434959100

Fu BX, St Onge RP, Fire AZ, Smith JD. 2016. Distinct patterns of Cas9 mismatch tolerance in vitro and in vivo. Nucleic Acids Res 44: 5365-5377. doi:10.1093/nar/gkw417

Hsu PD, Lander ES, Zhang F. 2014. Development and applications of CRISPR-Cas9 for genome engineering. Cell 157: 1262-1278. doi:10 .1016/j.cell.2014.05.010

Ishino Y, Shinagawa H, Makino K, Amemura M, Nakata A. 1987. Nucleotide sequence of the iap gene, responsible for alkaline phosphatase isozyme conversion in Escherichia coli, and identification of the gene product. $J$ Bacteriol 169: 5429-5433. doi:10.1128/JB.169.12.5429-5433.1987

Jiang WY, Bikard D, Cox D, Zhang F, Marraffini LA. 2013. RNA-guided editing of bacterial genomes using CRISPR-Cas systems. Nat Biotechnol 31: 233-239. doi:10.1038/nbt.2508

Jinek M, Chylinski K, Fonfara I, Hauer M, Doudna JA, Charpentier E. 2012. A programmable dual-RNA-guided DNA endonuclease in adaptive bacterial immunity. Science 337: 816-821. doi:10.1126/science.1225829

Koonin EV, Makarova KS, Zhang F. 2017. Diversity, classification and evolution of CRISPR-Cas systems. Curr Opin Microbiol 37: 67-78. doi:10.1016/ j.mib.2017.05.008

Le Rhun A, Escalera-Maurer A, Bratovič M, Charpentier E. 2019. CRISPR-Cas in Streptococcus pyogenes. RNA Biol 16: 380-389. doi:10.1080/15476286 .2019.1582974
Liang X, Potter J, Kumar S, Ravinder N, Chesnut JD. 2017. Enhanced CRISPR/Cas9-mediated precise genome editing by improved design and delivery of gRNA, Cas9 nuclease, and donor DNA. J Biotechnol 241: $136-146$. doi:10.1016/j.jbiotec.2016.11.011

Lin YN, Cradick TJ, Brown MT, Deshmukh H, Ranjan P, Sarode N, Wile BM, Vertino PM, Stewart FJ, Bao G. 2014. CRISPR/Cas9 systems have off-target activity with insertions or deletions between target DNA and guide RNA sequences. Nucleic Acids Res 42: 7473-7485. doi:10.1093/nar/ gku402

Makarova KS, Wolf YI, Alkhnbashi OS, Costa F, Shah SA, Saunders SJ, Barrangou R, Brouns SJ, Charpentier E, Haft DH, et al. 2015. An updated evolutionary classification of CRISPR-Cas systems. Nat Rev Microbiol 13: 722-736. doi:10.1038/nrmicro3569

Makarova KS, Wolf YI, Koonin EV. 2018. Classification and nomenclature of CRISPR-Cas systems: where from here? CRISPR J 1: 325-336. doi:10 .1089/crispr.2018.0033

Mojica FJ, Díez-Villaseñor C, Soria E, Juez G. 2000. Biological significance of a family of regularly spaced repeats in the genomes of Archaea, Bacteria and mitochondria. Mol Microbiol 36: 244-246. doi:10.1046/j.1365-2958 .2000.01838.x

Mojica FJ, Díez-Villaseñor C, García-Martínez J, Soria E. 2005. Intervening sequences of regularly spaced prokaryotic repeats derive from foreign genetic elements. J Mol Evol 60: 174-182. doi:10.1007/s00239-004-0046-3

Moon SB, Kim DY, Ko JH, Kim JS, Kim YS. 2019. Improving CRISPR genome editing by engineering guide RNAs. Trends Biotechnol 37: 870-881. doi:10.1016/j.tibtech.2019.01.009

Mougiakos I, Bosma EF, de Vos WM, van Kranenburg R, van der Oost J. 2016. Next generation prokaryotic engineering: the CRISPR-Cas toolkit. Trends Biotechnol 34: 575-587. doi:10.1016/j.tibtech.2016.02.004

Pyne ME, Moo-Young M, Chung DA, Chou CP. 2015. Coupling the CRISPR/ Cas9 system with lambda Red recombineering enables simplified chromosomal gene replacement in Escherichia coli. Appl Environ Microbiol 81: 5103-5114. doi:10.1128/AEM.01248-15

Reisch CR, Prather KL. 2015. The no-SCAR (Scarless Cas9 Assisted Recombineering) system for genome editing in Escherichia coli. Sci Rep 5: 15096 . doi:10.1038/srep15096

Ronda C, Pedersen LE, Sommer MO, Nielsen AT. 2016. CRMAGE: CRISPR optimized MAGE recombineering. Sci Rep 6: 19452. doi:10.1038/ srep19452

Sander JD, Joung JK. 2014. CRISPR-Cas systems for editing, regulating and targeting genomes. Nat Biotechnol 32: 347-355. doi:10.1038/nbt.2842

Sternberg SH, Doudna JA. 2015. Expanding the biologist's toolkit with CRISPR-Cas9. Mol Cell 58: 568-574. doi:10.1016/j.molcel.2015.02.032

Varani G, McClain WH. 2000. The G.U wobble base pair: a fundamental building block of RNA structure crucial to RNA function in diverse biological systems. EMBO Rep 1: 18-23. doi:10.1093/embo-reports/kvd001

Vento JM, Crook N, Beisel CL. 2019. Barriers to genome editing with CRISPR in bacteria. I Ind Microbiol Biotechnol 46: 1327-1341. doi:10.1007/ s10295-019-02195-1

Westra ER, Buckling A, Fineran PC. 2014. CRISPR-Cas systems: beyond adaptive immunity. Nat Rev Microbiol 12: 317-326. doi:10.1038/ nrmicro3241

Received September 25, 2019; accepted in revised form April 21, 2020. 


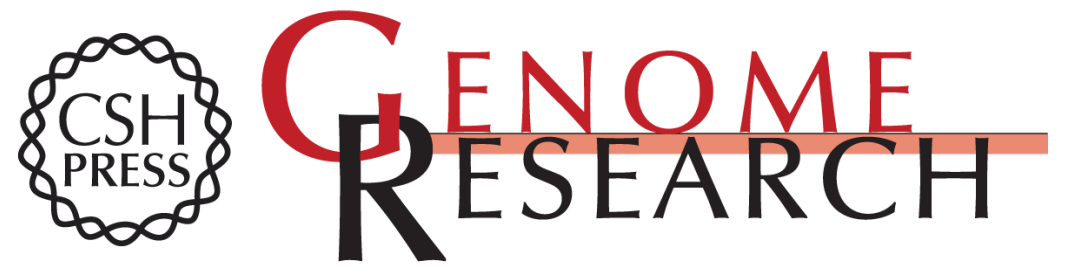

\section{CRISPR-Cas9-mediated pinpoint microbial genome editing aided by target-mismatched sgRNAs}

Ho Joung Lee, Hyun Ju Kim and Sang Jun Lee

Genome Res. 2020 30: 768-775 originally published online April 23, 2020

Access the most recent version at doi:10.1101/gr.257493.119

Supplemental Material

References

Open Access

Creative Commons

License

Email Alerting Service
http://genome.cshlp.org/content/suppl/2020/05/19/gr.257493.119.DC1

This article cites 29 articles, 5 of which can be accessed free at: http://genome.cshlp.org/content/30/5/768.full.html\#ref-list-1

Freely available online through the Genome Research Open Access option.

This article, published in Genome Research, is available under a Creative Commons License (Attribution 4.0 International), as described at http://creativecommons.org/licenses/by/4.0/.

Receive free email alerts when new articles cite this article - sign up in the box at the top right corner of the article or click here.

\section{Affordable, Accurate Sequencing.}

